\title{
Effects of Dielectric Environment on Phase Resonance in Compound Grating
}

\author{
Y. Xiao, ${ }^{1}$ Z. M. Liu, ${ }^{1}$ F. Q. Zhou, ${ }^{1}$ and H. J. Li $^{2}$ \\ ${ }^{1}$ College of Sciences, East China Jiaotong University, Nanchang 330013, China \\ ${ }^{2}$ College of Physics Science and Technology, Central South University, Changsha 410083, China \\ Correspondence should be addressed to Z. M. Liu; liuzhimin2006@163.com
}

Received 24 March 2015; Revised 6 July 2015; Accepted 12 July 2015

Academic Editor: Mihai V. Putz

Copyright (c) 2015 Y. Xiao et al. This is an open access article distributed under the Creative Commons Attribution License, which permits unrestricted use, distribution, and reproduction in any medium, provided the original work is properly cited.

\begin{abstract}
Effects of dielectric environment on phase resonance in compound grating are investigated theoretically and numerically. Transmission characteristics can be effectively tailored by changing the dielectric environment. When the dielectric constants between the two slits are slightly different, the transmission spectra show obvious dips for all modes, and values of the dips are near to zero, and photon forbidden band gaps emerge with larger difference of dielectric environment. The corresponding physical mechanisms are discussed by means of effective refractive index and effective length based on F-P-like and phase resonant mechanisms. In addition, the electric field for the peak at the shorter wavelength of the dip is mainly concentrated on the slit filled with smaller dielectric constant, while the electric field located on the slit filled with larger dielectric constant for the peak at the longer wavelength of the dip. By selecting a proper dielectric environment, the channel selecting device can be constructed by using the dielectric compound grating.
\end{abstract}

\section{Introduction}

Extraordinary optical transmission (EOT) has attracted great interest since the observation of EOT through a thick metal film perforated with a subwavelength holes array by Ebbesen et al. in 1998 [1]. Recently, the phase resonances in compound metallic nanostructures have aroused much attention, which result from phase difference and interference between adjacent slits or structures. The phase resonant dips in the transmission peak were demonstrated by theoretical calculation based on modal expansion theory [24]. Skigin experimentally confirmed the phase resonances in the millimeter-wave range by studying metallic periodic structures which comprised several subwavelength slits in each period $[5,6]$. Rance explored the phase resonances on metal gratings of identical, equally spaced, and alternately tapered slits in microwave regime [7].

Very recently, some methods have been presented to construct compound structure in order to achieve the phase resonances. For example, some authors built the compound metallic nanostructures from the perspective of different structure or the particularity of structure. Wang et al. [8] set symmetrical perpendicular cut in subwavelength metallic slits; He et al. [9] put a bar into the metallic slit; Zhai et al. [10] also introduced perpendicular cuts into the compound metallic grating; Fu et al. [11] investigated the effects of a bar on optical transmission through Z-shaped metallic slit arrays; Wu et al. [12] discussed the reflection and absorption spectra in compound metallic grating. In our previous paper [13], we proposed a compound metallic grating with perpendicular bumps and cuts in the slit. In addition, compound metallic nanostructures also have been achieved by compound gold surface relief slit arrays [14] and a gold grating with semicircle bumps [15]. The results of above papers showed that the influences of cuts or bars and bumps in the slit on odd and even modes of slit are different, which lead to the change of the effective length of the slits and phase difference, so the dips in transmission spectrum (namely, the phase resonances) will arise.

Actually, the transmission behaviors and phase resonance can be effectively tailored by changing the geometrical parameters and dielectric materials filled in the holes $[16,17]$. Another way to achieve compound metallic nanostructures has been used by adjusting dielectric environment [17] 
Xiang et al. [18] investigated the transmission resonances of compound metallic gratings with two subwavelength slits filled with different dielectrics inside each period, and they discussed the phase resonance from the different orders of F-P-like modes and found that when suitable dielectrics are chosen one is the Nth-order FP-like mode and the other is the $(N+1)$ th-order FP-like mode inside the two kinds of slits at a certain resonant frequency.

However, the phase resonances achieved by dielectric environment have not been studied comprehensively. In addition, the formation and evolution mechanisms of phase resonant dips and transmission spectra for all F-P-like modes are not investigated so far. In this paper, we try to comprehensively consider the effects of dielectric environment on the phase resonances which were investigated comprehensively in metallic gratings with two slits. Transmission characteristics are obviously tuned by changing the dielectric environment; all modes show sharp dips in transmission spectrum, which can be effectively tailored by changing slightly difference of dielectric constants between the two slits. Values of the dips are near to zero, and photon forbidden band gaps arise on transmission spectra with larger difference of dielectric environment between the two slits. The corresponding physical mechanisms are presented by the use of electric field distributions, F-P-like resonance, and phase resonance mechanisms.

\section{Model and Method}

A unit cell of a compound metallic grating with different dielectric environment in two slits is shown in Figure 1. The length, slit width of the grating, period, and the distance between the two slits are fixed in the whole paper: $L=$ $1500 \mathrm{~nm}, w=100 \mathrm{~nm}, p=700 \mathrm{~nm}$, and $D=350 \mathrm{~nm}$. The dielectric constants are denoted by $\varepsilon_{1}$ and $\varepsilon_{2}$, respectively. And the metals are chosen to be gold ( $\mathrm{Au})$. The optical properties of the gold nanostructure are approximated by the Drude model and the dispersive permittivity of the frequency is defined as

$$
\varepsilon(\omega)=1-\frac{\omega_{p}^{2}}{\omega^{2}+\gamma_{p}^{2}}+i \frac{\omega_{p}^{2} \gamma_{p}^{2}}{\omega\left(\omega^{2}+\gamma_{p}^{2}\right)},
$$

where $\omega$ is the frequency of the incident light, $\omega_{p}=1.37 \times$ $10^{16} \mathrm{~s}^{-1}$, and $\gamma_{p}=4.08 \times 10^{13} \mathrm{~s}^{-1}$ represents the bulk plasmon frequency and the damping rate which characters the absorption loss [19].

We use two-dimensional FDTD $[20,21]$ method to calculate the transmission spectra and the time-dependent fields; the size of FDTD lattice is $L_{x} \times L_{y}=700 \mathrm{~nm} \times 2800 \mathrm{~nm}$; $\Delta_{x}=\Delta_{y}=1 \mathrm{~nm}$ and $\Delta t=\Delta x / 2 c$ are the spatial and temporal steps. Boundary condition on the top and bottom boundaries of the unit cell along $y$ direction is truncated by perfectly matched layer absorbing boundary conditions which is treated by periodic boundary conditions on the left and right boundaries along $x$ direction which are shown in Figure 1 . The incident light spreads through $y$ direction with TM polarization.

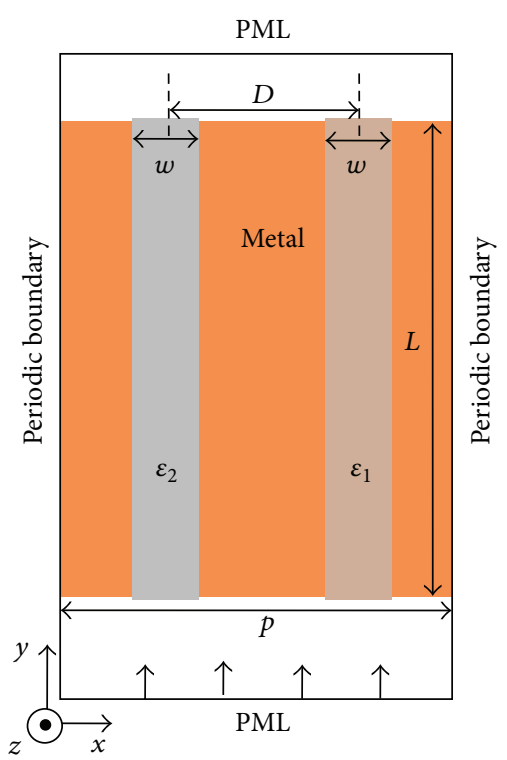

FIGURE 1: Scheme of a unit cell of the grating with different dielectric environment in two slits.

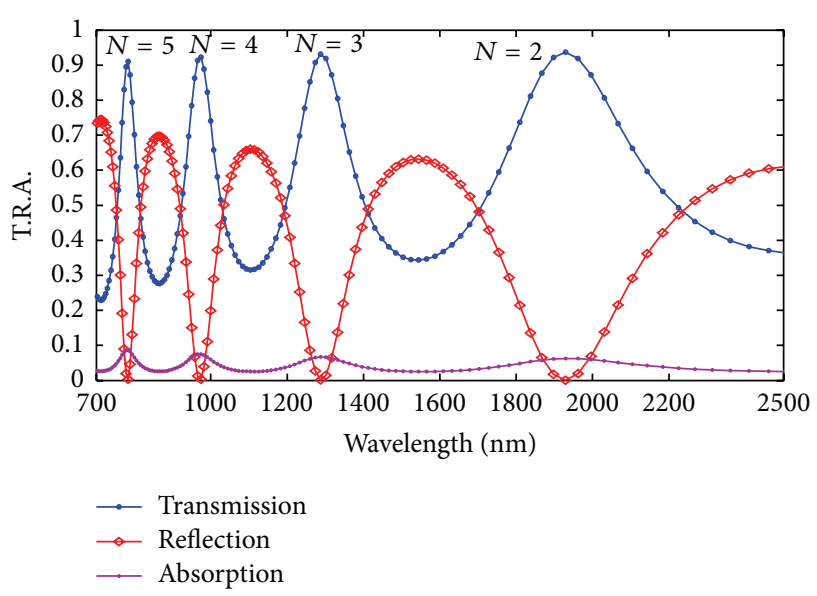

Figure 2: Transmission, reflection, and absorption spectra for two slits with same dielectric constants.

\section{Results and Discussion}

Firstly, in order to better understand the effects of dielectric environment, the transmission, reflection, and absorption spectra of the grating with same dielectric constants $\left(\varepsilon_{1}=\right.$ $\varepsilon_{2}=1$ ) in two slits are shown in Figure 2. The probe location of transmission spectra is set at $300 \mathrm{~nm}$ away from the rear surface of the export, and the probe location of reflection spectra is set at $160 \mathrm{~nm}$ away from the front of surface. The calculated spectra are normalized by the calculation without a metallic structure. And the absorption spectra can be obtained by $\mathrm{A}=1-\mathrm{T}-\mathrm{R}$, where $\mathrm{T}, \mathrm{R}$, and $\mathrm{A}$ represent the transmittance, reflectance, and absorbance, respectively.

The transmission spectrum in Figure 2 (blue line) shows that the wavelengths of Fabry-Pérot-like modes resonant 


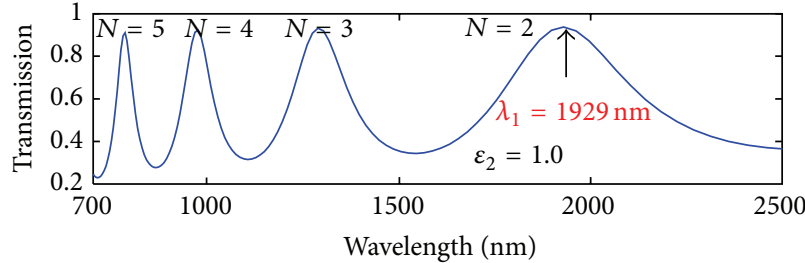

(a)

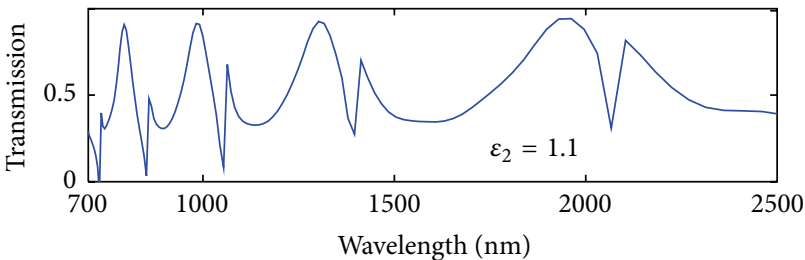

(c)

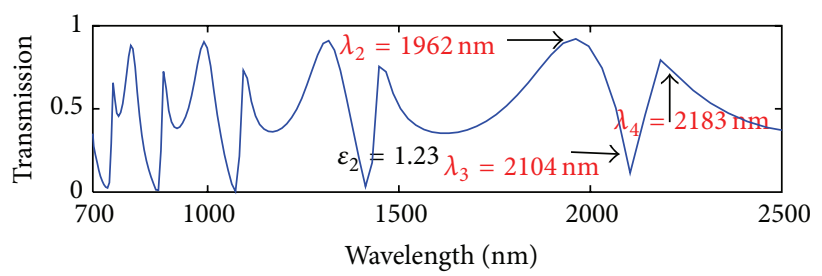

(e)

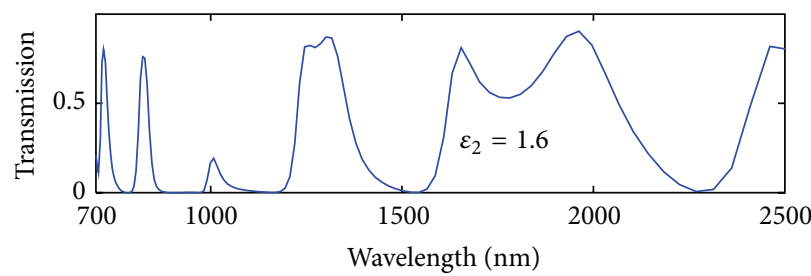

(g)

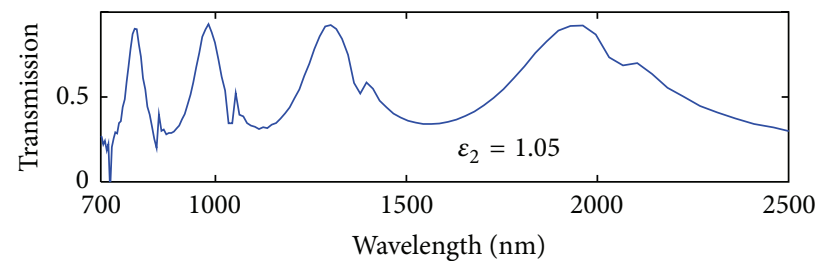

(b)

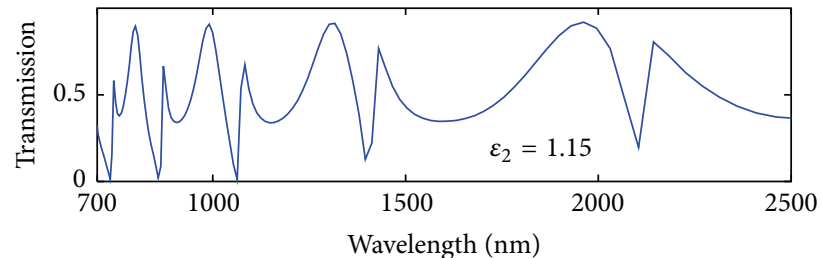

(d)

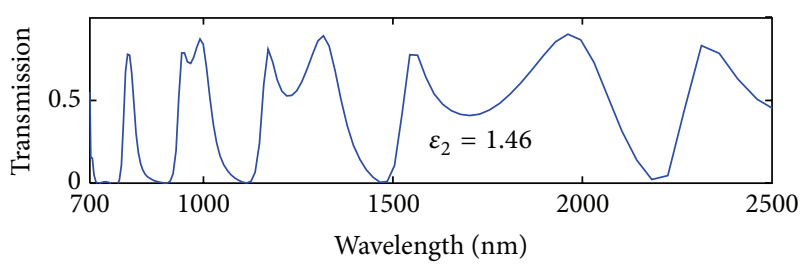

(f)

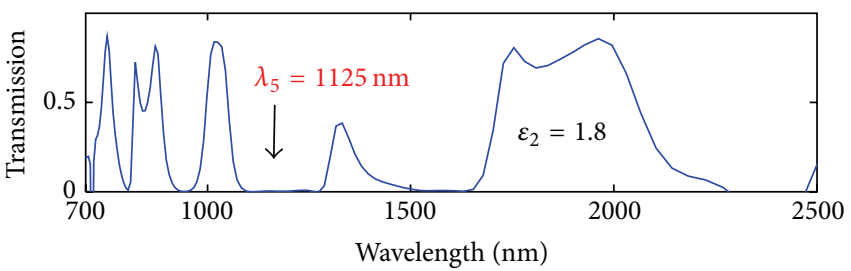

(h)

FIgURE 3: Transmission spectra as a function of wavelength for different dielectric constants in the two slits. The value of insets shows the dielectric constants.

peaks are $1929 \mathrm{~nm}, 1287 \mathrm{~nm}, 974 \mathrm{~nm}$, and $778 \mathrm{~nm}$, respectively, according to previously reported results $[8,13]$, which are labeled as $N=2, N=3, N=4$, and $N=5$, respectively. The resonant modes are associated with different standing wave modes in the slit. For example, if the resonant peak is labeled as $N=2$, two nodes (antinodes) of the electric (magnetic) field appear in the slit. We know that the Fabry-Pérot-like modes are characterized by constructive interference between all transmitted waves along the slit [22], and the condition of the F-P can be expressed as [23]

$$
k_{0} \operatorname{Re}\left(n_{\mathrm{eff}}\right) L_{\mathrm{FP}}+\arg (\rho)=n \pi,
$$

where $k_{0}=2 \pi / \lambda$ is the wave vector in free space, $n_{\text {eff }}$ is the effective refractive index in the slit, which strongly depends on the slit width, $\rho$ stands for the reflection coefficient of the fundamental mode, $L_{\mathrm{FP}}$ represents the slit length, and $n$ is integer.

In addition, red line in Figure 2 shows that there is strong reflection at the bottom of transmission. In other words, the largest transmission indicates the lowest reflectance; on the contrary, the strongest reflectance represents the weakest transmission. Next, purple line in Figure 2 presents very little absorption rate; the absorption spectra are obtained by $\mathrm{A}=$ $1-\mathrm{T}-\mathrm{R}$; this is obviously related to the absorption loss of light energy by metal gold.

In the previous section, we have presented the transmission, reflection, and absorption spectra of the grating with the same dielectric constant $\left(\varepsilon_{1}=\varepsilon_{2}=1\right)$ in two slits. Next, we study the effects of dielectric environment on the phase resonances and simulate the transmission spectrum for metallic grating with different dielectric environment in two slits, namely, the asymmetric case. In order to well understand effects of dielectric environment on the phase resonances, the dielectric constant in one of the slits is fixed as $\varepsilon_{1}=1$; the dielectric constant $\varepsilon_{2}$ of the other slit changes from 1 to 1.15 with increment 0.05 in Figures $3(\mathrm{a})-3(\mathrm{~d})$ and to 1.23, $1.46,1.6$, and 1.8 in Figures $3(\mathrm{e})-3(\mathrm{~h})$. We can find that all Fabry-Pérot-like mode peaks exhibit dips when $\varepsilon_{2}$ changes to 1.05 , which are shown in Figure 3(b), but the dips for modes $N=2, N=3, N=4$, and $N=5$ are smaller. 
With the increase of $\varepsilon_{2}$, the dips are further deepened. In our previous paper [13], we studied a compound metallic grating with perpendicular bumps and cuts in the slit; the results showed that the phase resonant dips can be tuned by shifting the position or changing the size of bumps and cuts. Now, let us make a comparison between the above results and previously reported results [13]. Perpendicular bumps and cuts only affect one or several modes when the bumps or cuts locate at the center of modes, which brings the most influence on those modes and the effective length of the F-P cavity. However, the bumps or cuts are not located at the center of modes, which affect modes slightly. In contrast, dielectric environment affects all modes because the dielectric medium is filled in the whole slits. So we can see that all Fabry-Pérotlike mode peaks exhibit dips.

When the dielectric constant changes to 1.23 and 1.46 , the dips are further deepened and almost all the values of the dips are near to zero. As the dielectric constant further varies to 1.6 and 1.8, forbidden band gaps appear on transmission spectra; in this case the propagation of the incident light will be stopped. Actually, the field can still be distributed in the two slits if we reduce the relative value of intensity in drawing the figures, but the wave cannot propagate over the two slits. The number of the forbidden gap increases and the forbidden gaps get broaden when difference between dielectric values in two slits becomes further larger. The reason for such behavior is that when difference between dielectric values in two slits becomes larger difference between the wavelengths of same order resonant peak in the two cases gets further larger, which results in the fact that the dips are further deepened and the forbidden gaps get broadened.

Based on above analysis, we can say that phase resonance is more sensitive to dielectric environment than other adjustable factors, such as bumps and cuts. The results show that an optical filter or frequency selector can be designed according to the adjustment of the dielectric constant.

The physical origin of the dips can be explained in terms of the theory as follows. The resonant wavelength of F-P mode in a bare slit array can be obtained by (2). In previous results [13], the bump and cut affect the surface charge and surface current flow on the slit, and then the total effective length of the F-P cavity is determined by

$$
L_{\text {eff }}=L_{\mathrm{FP}}+\Delta ;
$$

if $\Delta>0$, the effective length $L_{\text {eff }}$ increases and the resonant wavelength gets larger. When $\Delta<0$, the effective length $L_{\text {eff }}$ decreases, and then the resonant wavelength becomes smaller. From (2), we know that the resonant wavelength also can be adjusted by $n_{\text {eff }}$, and $n_{\text {eff }}$ is the effective refractive index which can be obtained by solving the dispersion equation [23]:

$$
\begin{aligned}
& \varepsilon_{m} \sqrt{n_{\mathrm{eff}}^{2}-\varepsilon_{d}} \tanh \left(\frac{w \pi \sqrt{n_{\mathrm{eff}}^{2}-\varepsilon_{d}}}{\lambda}\right)+\varepsilon_{d} \sqrt{n_{\mathrm{eff}}^{2}-\varepsilon_{d}} \\
& \quad=0
\end{aligned}
$$

where $w$ is the width of metal-dielectric-metal waveguide and $\varepsilon_{d}$ and $\varepsilon_{m}$ are permittivities for dielectric and metal, respectively. We notice that, for not too small gaps, $n_{\mathrm{eff}}$ can be approximated as follows:

$$
n_{\mathrm{eff}}=\frac{k_{\mathrm{MDM}}}{k_{0}} \approx \sqrt{\varepsilon_{d}-\frac{2 \varepsilon_{d} \sqrt{\varepsilon_{d}-\varepsilon_{m}}}{k_{0} w \varepsilon_{m}}},
$$

where $k_{0}=2 \pi / \lambda$ and $k_{\mathrm{MDM}}$ are the wave vector in free space and metal-dielectric-metal waveguide.

Based on (5), Figure 4(a) plots the variation of the real parts of $n_{\text {eff }}$ for the metallic slit filled with different dielectric $\varepsilon_{d}=1,1.23$ and 1.46 , respectively. The results show that effective refractive index $n_{\text {eff }}$ of the metal-dielectric-metal waveguide is always greater than that of the metal-air-metal waveguide for the same wavelength and slit width $w$, which can be seen more clearly from Figure 4(a); the corresponding wavelength is fixed $\lambda=1550 \mathrm{~nm}$. According to Figure 4(a), we could obtain $n_{\text {eff }}$ equaling to $1.222,1.315$, and 1.402 for $\varepsilon_{d}=1,1.23$ and 1.46 , respectively. Other parameters are set as follows: slit width is $w=100 \mathrm{~nm}$, and the wavelength for the dip labeled as $N=2$ is $2104 \mathrm{~nm}$, which is labeled in Figure 3(e). When the dielectric constant increases, $n_{\text {eff }}$ gets larger, and then the resonant wavelength shifts to longer region. In contrast, the resonant wavelength becomes smaller with decreasing of the dielectric constant. In the case where a light is normally incident when the dielectrics in two slits are the same $\varepsilon_{1}=\varepsilon_{2}$ the translation invariance can reduce the field degrees of freedom to just like a single periodic slit array. Therefore, the fields in all slits are equal. While the dielectrics in two slits are different $\varepsilon_{1} \neq \varepsilon_{2}$ the fields of two slits are not identical, which shows that there exists phase difference; it can also be understood that the field phases in two slits are unequal $[4,10,24]$. When the phase difference between adjacent slits reaches a certain value, an obvious dip appears which results from destructive interference between two slits. Figure 4(b) testifies our above explanation. All the mode peaks in the transmission spectrum show red-shift when the refractive index of dielectric increases and the numbers of the resonant mode increase. For example, considering wave range from visible light to near infrared band, six, seven, and eight mode peaks in the transmission spectrum appear for the case where $\varepsilon_{d}=1,1.23$ and 1.46 , respectively. The effective refractive index $n_{\text {eff }}$ of the metal-dielectric-metal increases with the increase of dielectric constant; it is equal to the increase of effective length of the metal-air-metal.

Then we analyze quantitatively the above behavior. When the dielectric constants in two slits are equal $\left(\varepsilon_{1}=\varepsilon_{2}=1\right)$, the corresponding wavelength of resonant peak for $N=2$ mode is $1929 \mathrm{~nm}$; and when the dielectric constants in two slits are equal to $\left(\varepsilon_{1}=\varepsilon_{2}=1.23\right)$, the corresponding wavelength is $2143 \mathrm{~nm}$, which is shown in Figure 4(b). While the dielectrics filling the two slits are different, for example, one is $\varepsilon_{1}=1$ and the other is 1.23 , an obvious dip appears due to phase resonance. The corresponding wavelengths of the two resonance peaks at both sides of dip are $1962 \mathrm{~nm}$ and $2183 \mathrm{~nm}$, which are close to $1929 \mathrm{~nm}$ (for $\varepsilon_{1}=\varepsilon_{2}=1$ ) and $2143 \mathrm{~nm}$ (for $\varepsilon_{1}=\varepsilon_{2}=1.23$ ), respectively. This phenomenon can be quite acceptable and understood as a superposition of two resonant modes. 


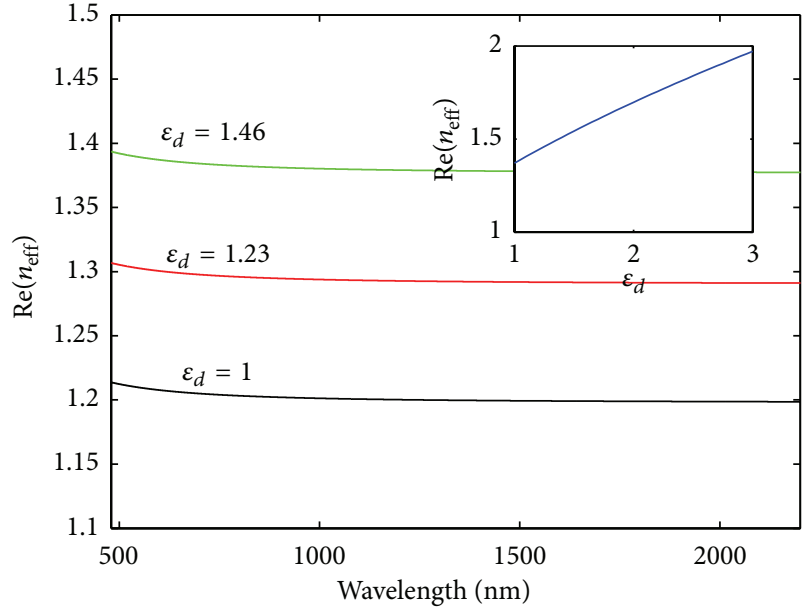

(a)

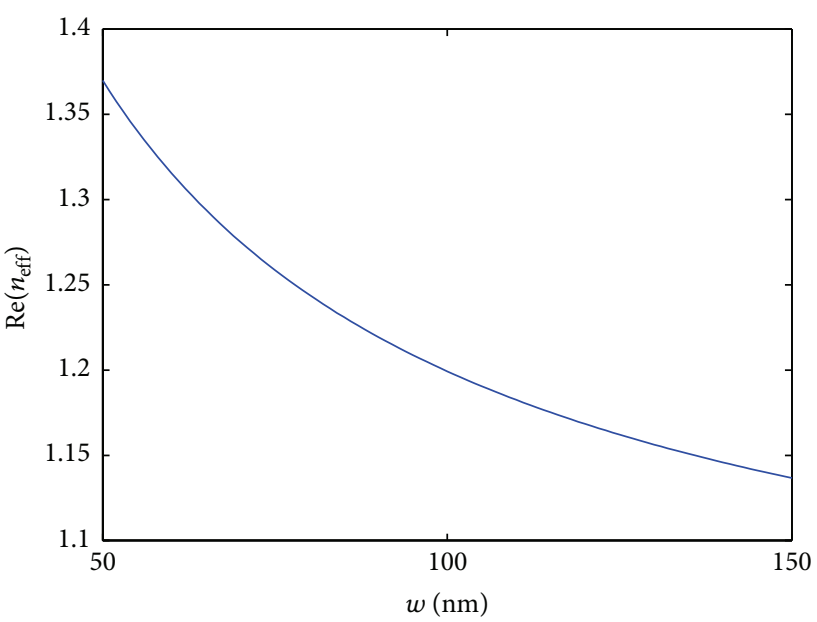

(c)

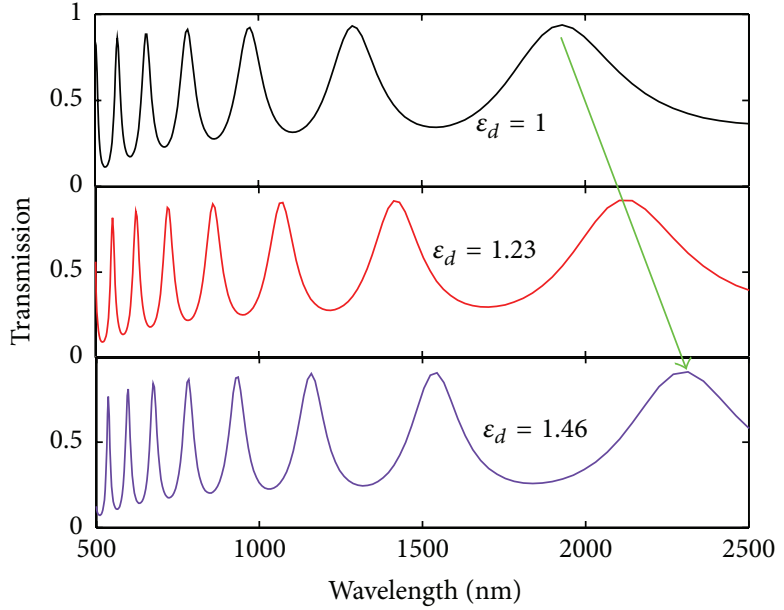

(b)

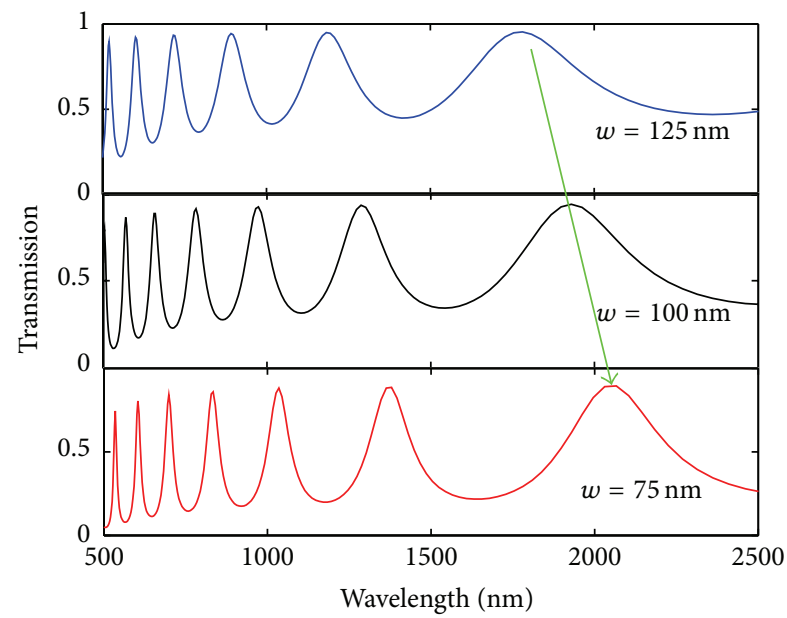

(d)

FIGURE 4: (a) Real part of the effective refractive index $n_{\text {eff }}$ as a function of wavelength for different $\varepsilon_{d}$. (b) Transmission spectra as a function of wavelength for different $\varepsilon_{d}$ for the same dielectric environment in two slits (namely, single slit). (c) Real part of $n_{\text {eff }}$ as slit width $w$. (d) Transmission spectra as a function of wavelength for different slit width $w$ for single slit.

Figure 4(c) displays the dependence of $n_{\text {eff }}$ on the slit width at $\lambda=1.55 \mu \mathrm{m}$. It is clear that the real parts of $n_{\text {eff }}$ decrease with the increase of slit widths; different slit widths lead to the difference of effective refractive indices. The conclusions are also confirmed by Figure 4(d), which shows the transmission spectra as a function of wavelength for different slit width $w$ for single slit. With the decrease of slit width $w$, all the transmission mode peaks in the transmission spectrum have red-shift; the numbers of transmission mode peaks also increase in the same wavelength range.

Now, let us make a comparison with the dependence of dielectric environment and slit width on effective refractive index and effective length. Both of them affect effective refractive index or effective length, but it should be noted that increase of the dielectric constant results in an increase of the effective refractive index or the effective length, while increase of slit width leads to a decrease of the effective refractive index or the effective length. Furthermore, it is obviously sensitive to the dielectric environment; it can be seen from Figures $4(\mathrm{~b})-4(\mathrm{~d})$.

To understand the effects of dielectric environment on the phase resonances well, in Figure 5, we draw the electric field distributions for different wavelength: (a) $\lambda_{1}=1929 \mathrm{~nm}$, (b) $\lambda_{2}=1962 \mathrm{~nm}$, (c) $\lambda_{3}=2104 \mathrm{~nm}$, (d) $\lambda_{4}=2183 \mathrm{~nm}$, and (e) $\lambda_{5}=1125 \mathrm{~nm}$, which are labeled in Figure 3, respectively. We can see that Fabry-Pérot-like modes distribute inside each slit (the same as single slit) along the longitudinal direction for the resonance mode peak $N=2$ in Figure 5(a). Meanwhile, in Figure 5(b), we find that the electric field for the resonant peak at the shorter wavelength $\lambda_{2}=1962 \mathrm{~nm}$ near the left of dip is mainly concentrated on the right slit filled with smaller dielectric constant and the electric field located on the left slit filled with larger dielectric constant for the resonant peak at the longer wavelength $\lambda_{4}=2183 \mathrm{~nm}$ near the right of dip; the results are shown in Figure 5(d); the corresponding wavelength of the dip is $\lambda_{3}=2104 \mathrm{~nm}$. The reasons of 


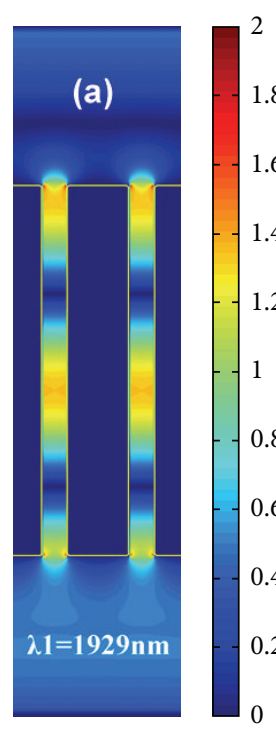

(a)

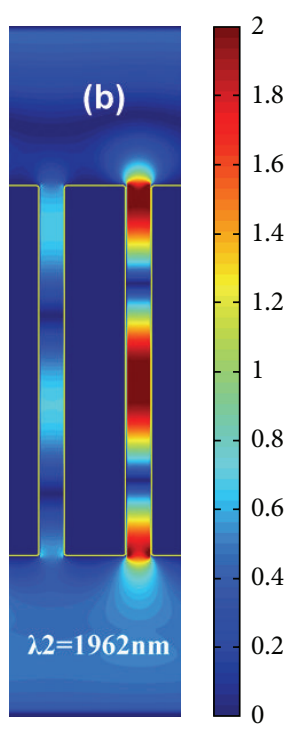

(b)

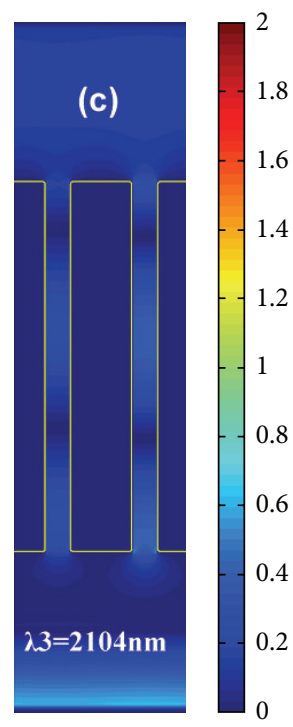

(c)

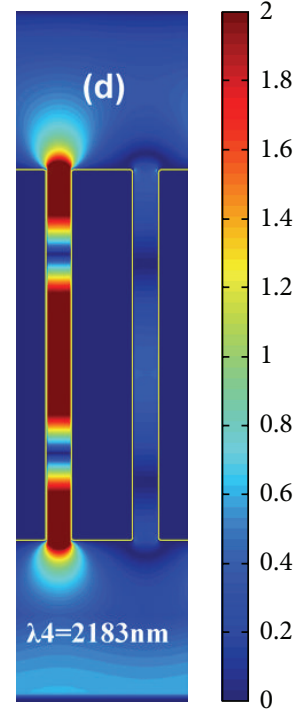

(d)

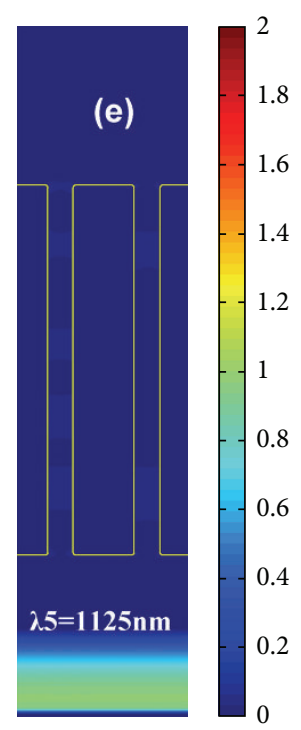

(e)

FIGURE 5: The magnitudes of electric field $\left|E_{x}\right|$ of compound gratings for different wavelength, (a) $\lambda_{1}=1929 \mathrm{~nm}$, (b) $\lambda_{2}=1962 \mathrm{~nm},(\mathrm{c})$ $\lambda_{3}=2104 \mathrm{~nm}$, (d) $\lambda_{4}=2183 \mathrm{~nm}$, and (e) $\lambda_{5}=1125 \mathrm{~nm}$, which are labeled in Figure 3.

the phenomenon can be explained as follows. The shorter wavelength $\lambda_{2}=1962 \mathrm{~nm}$ near the left of dip (labeled in Figure 3) for the case of different dielectric constants is close to the wavelength of F-P mode resonant peaks $1929 \mathrm{~nm}$, which is the case of the equal and smaller dielectric constants $\left(\varepsilon_{1}=\varepsilon_{2}=1\right)$ in two slits (marked in Figure 2). Then the electric field for the resonant peak at the shorter wavelength $\lambda_{2}=1962 \mathrm{~nm}$ is mainly concentrated on the right slit. In other words, the resonant transmission mainly depends on right slit filled with smaller dielectric constant. On the other hand, when the dielectric constants in two slits are equal to $\varepsilon_{1}=\varepsilon_{2}=1.23$, the wavelength of Fabry-Pérot-like mode resonant peaks is $2143 \mathrm{~nm}$ (marked in Figure 4(c)), which is close to the larger wavelength $\lambda_{2}=2183 \mathrm{~nm}$ near the right of dip (labeled in Figure 3) for the case of different dielectric constants, and then the electric field for the peak at the larger wavelength $\lambda_{2}=2183 \mathrm{~nm}$ is mainly concentrated on the left slit filled with larger dielectric constant.

Compared with Figures 5(a), 5(b), and 5(d), the electric field distribution is very weak for wavelength $\lambda_{3}=2104 \mathrm{~nm}$ of the dip when we draw the figures in the same standard of intensity; the result is shown in Figure 5(c), which proves that the dip is low transmission. In addition, in Figure 5(e), we choose the wavelength $\lambda_{5}=1125 \mathrm{~nm}$ in forbidden band gaps on transmission spectra; the result shows that the electric field distribution is weaker. Actually the electric field can still be distributed in the two slits if we reduce the relative value intensity in drawing the figures, but the wave cannot propagate over the two slits; the result also presents strongly the reflection feature.

The above results confirm that the interference behavior of phase resonances between adjacent slits can be modulated by dielectric environment of compound grating; an optical filter and channel selecting devices can be constructed by selecting appropriate dielectric environment based on a compound metallic grating.

\section{Conclusion}

In this paper, we have theoretically investigated the phase resonances of compound metallic gratings with two subwavelength slits filled with different dielectric environments. For comparison, the transmission, reflection, and absorption spectra of the slits with the same dielectric constants are presented and discussed. In addition, we find that phase resonant characteristics can be more effectively tailored by changing the dielectric environment than by other adjustable factors. Slight difference of dielectric constants between the two slits may bring obvious dips; almost all the values of the dips are near to zero, and photon forbidden band gaps appear on transmission spectra with larger difference of dielectric environment. The corresponding physical mechanisms for above phenomenon are discussed by use of electric field distributions, F-P-like resonance, and phase resonance mechanisms. Based on the electric field distributions, we can find that the electric field for the peak at the shorter wavelength is mainly concentrated on the slit filled with smaller dielectric constant, while the electric field located on the slit filled with larger dielectric constant for the peak at the longer wavelength. By selecting appropriate dielectric environment, an optical filter and channel selecting devices can be constructed based on a compound metallic grating. These results are useful for the design of the nanooptic devices and may contribute to more real applications in the future.

\section{Conflict of Interests}

The authors declare that there is no conflict of interests regarding the publication of this paper. 


\section{Acknowledgments}

This work was funded by the National Natural Science Foundation of China (nos. 11164007, 61275174, 61107055, and 11364019), the Natural Science Foundation of Jiangxi Province (no. 20132BAB212007), the Educational Reform Project of Jiangxi Province (no. JXJG-14-5-25), and the Project of East China Jiaotong University (14LX06).

\section{References}

[1] T. W. Ebbesen, H. J. Lezec, H. F. Ghaemi, T. Thio, and P. A. Wolff, "Extraordinary optical transmission through sub-wavelenght hole arrays," Nature, vol. 391, no. 6668, pp. 667-669, 1998.

[2] J. Q. Liu, M. D. He, X. Zhai, and L. L. Wang, "Tailoring optical transmission via the arrangement of compound subwavelength hole arrays," Optics Express, vol. 17, no. 3, pp. 1859-1864, 2009.

[3] J. Q. Liu, X. B. Chao, J. N. Wei et al., "Multiple enhanced transmission bands through compound periodic array of rectangular holes," Journal of Applied Physics, vol. 106, no. 9, Article ID 093108, 2009.

[4] Z. F. Liu and G. J. Jin, "Phase effects in the enhanced transmission through compound subwavelength rectangular hole arrays," Journal of Applied Physics, vol. 106, no. 6, Article ID 063122, 2009.

[5] D. C. Skigin and R. A. Depine, "Transmission resonances of metallic compound gratings with subwavelength slits," Physical Review Letters, vol. 95, no. 21, Article ID 217402, 2005.

[6] M. Navarro-Cía, D. C. Skigin, M. Beruete, and M. Sorolla, "Experimental demonstration of phase resonances in metallic compound gratings with subwavelength slits in the millimeter wave regime," Applied Physics Letters, vol. 94, no. 9, 2009.

[7] H. J. Rance, O. K. Hamilton, J. R. Sambles, and A. P. Hibbins, "Phase resonances on metal gratings of identical, equally spaced alternately tapered slits," Applied Physics Letters, vol. 95, no. 4, Article ID 041905, 2009.

[8] Y. Wang, Y. Wang, Y. Zhang, and S. Liu, "Transmission through metallic array slits with perpendicular cuts," Optics Express, vol. 17, no. 7, pp. 5014-5022, 2009.

[9] M. D. He, Z. Q. Gong, S. Li, Y. F. Luo, J. Q. Liu, and X. Chen, "Light transmission through metallic slit with a bar," Solid State Communications, vol. 150, no. 29-30, pp. 1283-1286, 2010.

[10] X. Zhai, J. Q. Liu, M. D. He, L. L. Wang, S. Wen, and D. Fan, "Adjustable phase resonances in a compound metallic grating with perpendicular cuts," Optics Express, vol. 18, no. 7, pp. 68716876, 2010.

[11] Z. P. Fu, F. Lin, and X. Zhu, "Numerical study on the optical absorption of one dimension metallic gratings," Acta Physica Sinica, vol. 60, no. 11, Article ID 114213, 2011.

[12] C. Wu, H. Li, X. Peng, G. Cao, and Z. Liu, "Effects of a bar on optical transmission through Z-shaped metallic slit arrays," Chinese Physics B, vol. 22, no. 5, Article ID 057301, 2013.

[13] Z. M. Liu, H. J. Li, S. X. Xie et al., "Tunable phase resonances in a compound metallic grating with perpendicular bumps and cuts," Optics Express, vol. 19, no. 5, pp. 4217-4222, 2011.

[14] X. Zhou, J. S. Fang, D. W. Yang, X. Zhao, B. Tang, and Z. M. Liu, "Optical transmission through compound gold surface relief slit arrays," Optics Express, vol. 22, no. 1, pp. 1085-1093, 2014.

[15] X. Zhou, J. Fang, Q. Zhu, B. Tang, and Z. Liu, "Investigation of optical transmission through a gold grating with semicircle bumps using FDTD method," Modern Physics Letters B, vol. 27, no. 17, Article ID 1350126, 2013.

[16] X. N. Zhang, G. Q. Liu, Y. Hu et al., “Tunable extraordinary optical transmission in a metal film perforated with two-level subwavelength cylindrical holes," Plasmonics, vol. 9, no. 5, pp. 1149-1153, 2014.

[17] D. C. Skigin, H. Loui, Z. Popovic, and E. F. Kuester, "Bandwidth control of forbidden transmission gaps in compound structures with subwavelength slits," Physical Review E-Statistical, Nonlinear, and Soft Matter Physics, vol. 76, no. 1, Article ID 016604, 2007.

[18] D. Xiang, L. L. Wang, X. F. Li et al., "Transmission resonances of compound metallic gratings with two subwavelength slits in each period," Optics Express, vol. 19, no. 3, pp. 2187-2192, 2011.

[19] A. Taflove and S. C. Hagness, Computational Electrodynamics: The Finite-Difference Time-Domain Method, Artech House, Boston, Mass, USA, 2000.

[20] K. S. Yee, "Numerical solution of inital boundary value problems involving Maxwell's equations in isotropic media," IEEE Transactions on Antennas and Propagation, vol. 14, no. 3, pp. 302-307, 1966.

[21] E. D. Palik, Handbook of Optical Constants in Solids, Academic Press, Boston, Mass, USA, 1982.

[22] A. P. Hibbins, M. J. Lockyear, and J. R. Sambles, "The resonant electromagnetic fields of an array of metallic slits acting as Fabry-Perot cavities," Journal of Applied Physics, vol. 99, no. 12, Article ID 124903, 2006.

[23] P. Lalanne, C. Sauvan, J. P. Hugonin, J. C. Rodier, and P. Chavel, "Perturbative approach for surface plasmon effects on flat interfaces periodically corrugated by subwavelength apertures," Physical Review B-Condensed Matter and Materials Physics, vol. 68, no. 12, Article ID 125404, 2003.

[24] Z. Liu and G. J. Jin, "Resonant acoustic transmission through compound subwavelength hole arrays: the role of phase resonances," Journal of Physics: Condensed Matter, vol. 21, no. 44, Article ID 445401, 2009. 

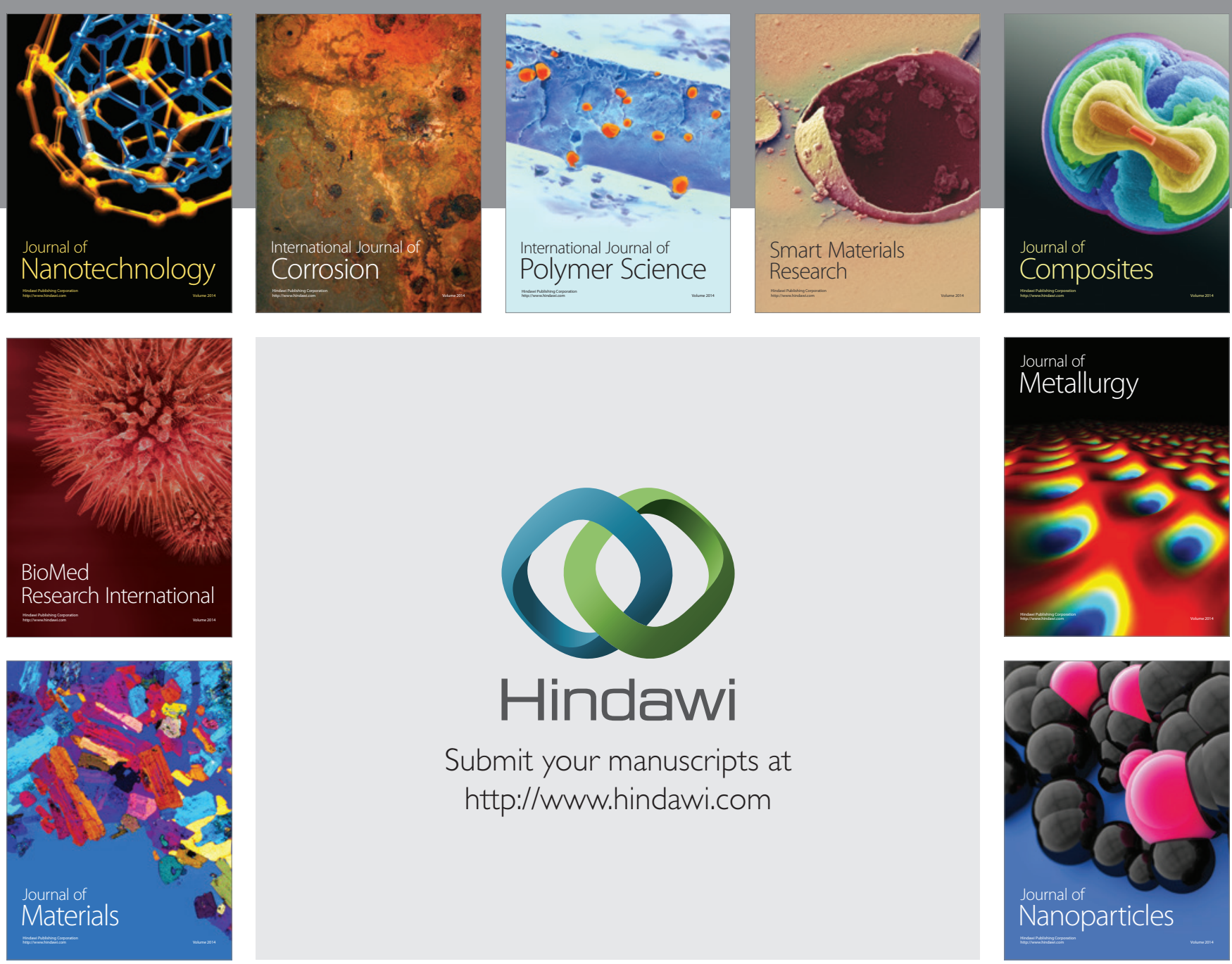

Submit your manuscripts at http://www.hindawi.com
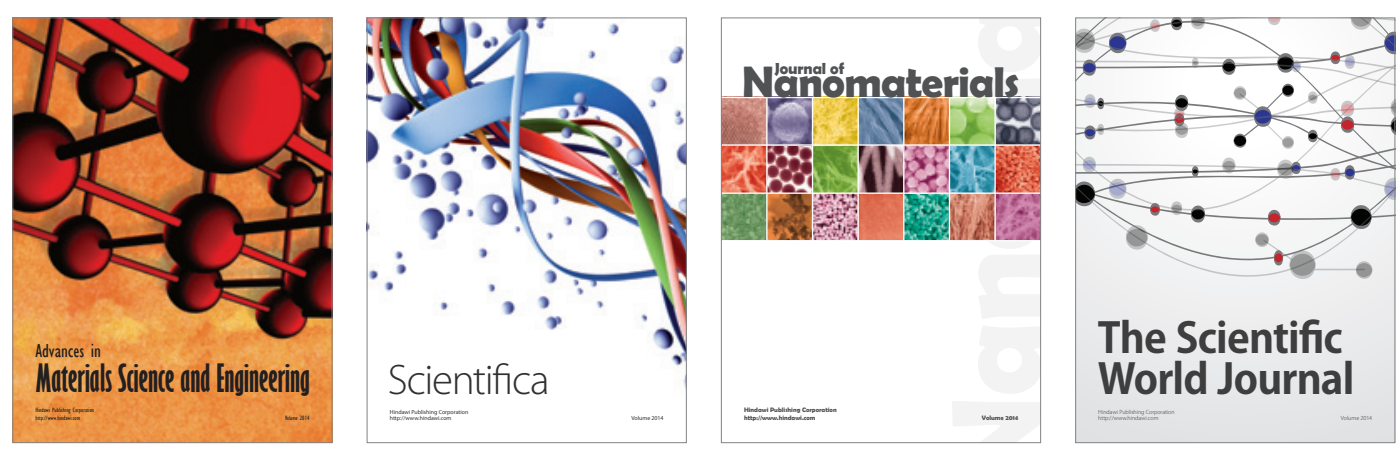

\section{The Scientific World Journal}
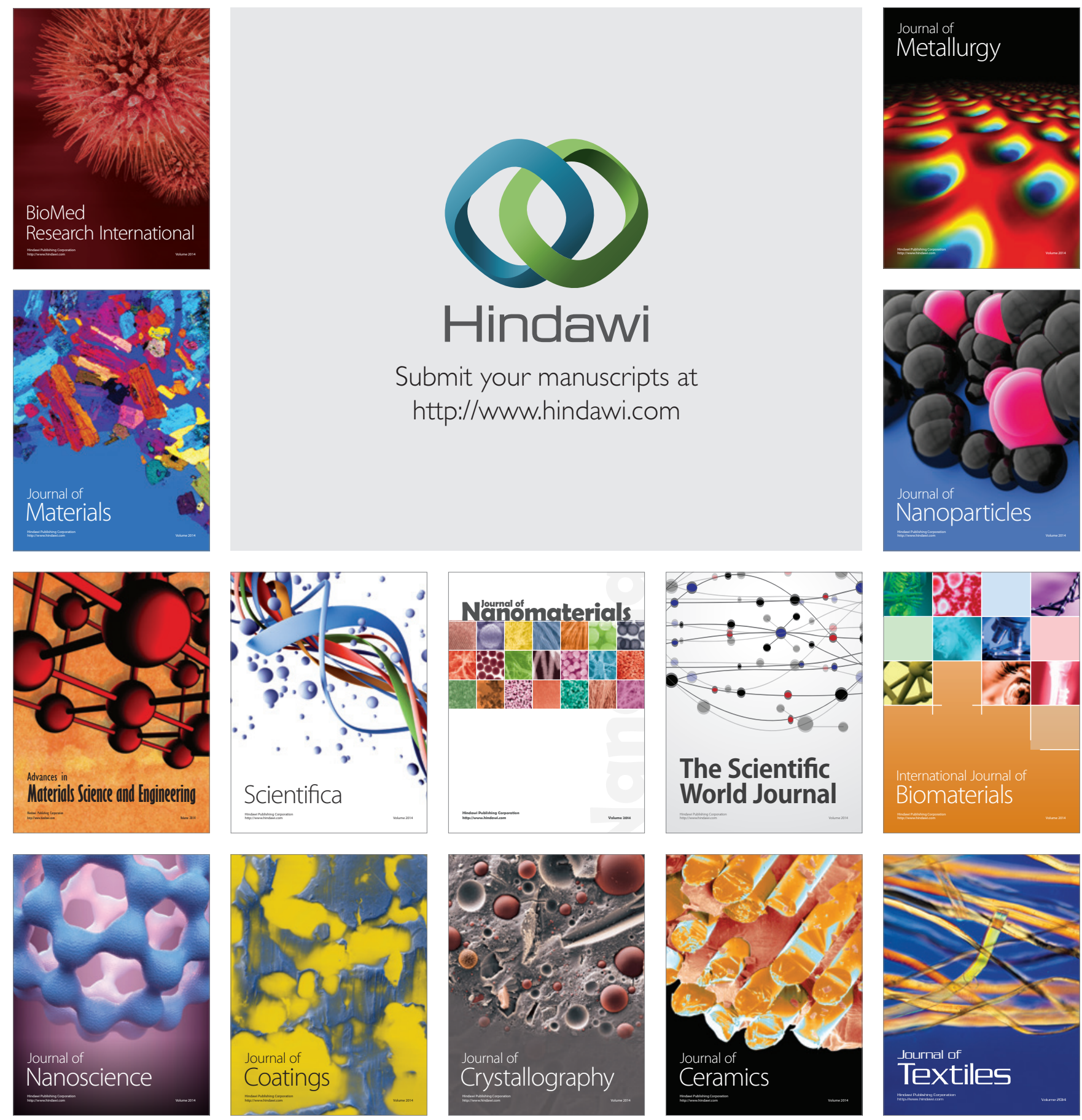